\title{
Transport in a Lévy ratchet: Group velocity and distribution spread
}

\author{
B. Dybied* and E. Gudowska-Nowak $\AA^{*}$ \\ M. Smoluchowski Institute of Physics, and Mark Kac Center for Complex Systems Research, \\ Jagellonian University, ul. Reymonta 4, 30-059 Kraków, Poland \\ I. M. Sokolov \\ Institut für Physik, Humboldt-Universität zu Berlin, Newtonstrasse 15, D-12489 Berlin, Germany
}

(Dated: December 1, 2018)

\begin{abstract}
We consider the motion of an overdamped particle in a periodic potential lacking spatial symmetry under the influence of symmetric Lévy noise, being a minimal setup for a "Lévy ratchet." Due to the non-thermal character of the Lévy noise, the particle exhibits a motion with a preferred direction even in the absence of whatever additional time-dependent forces. The examination of the Lévy ratchet has to be based on the characteristics of directionality which are different from typically used measures like mean current and the dispersion of particles' positions, since these get inappropriate when the moments of the noise diverge. To overcome this problem, we discuss robust measures of directionality of transport like the position of the median of the particles displacements' distribution characterizing the group velocity, and the interquantile distance giving the measure of the distributions' width. Moreover, we analyze the behavior of splitting probabilities for leaving an interval of a given length unveiling qualitative differences between the noises with Lévy indices below and above unity. Finally, we inspect the problem of the first escape from an interval of given length revealing independence of exit times on the structure of the potential.
\end{abstract}

PACS numbers: 05.40.Fb, 05.10.Gg, 02.50.-r, 02.50.Ey,

\section{INTRODUCTION}

Motion of particles in an external periodic potential with broken spatial symmetry and under influence of external forces with zero mean can result in occurrence of the persistent, directed current [1, 2, 3, 4, 5]. The occurrence of the directed transport is a consequence of the violation of the detailed balance in systems acting away from equilibrium. Typically, one assumes that the overall force acting on the particle is a superposition of the Gaussian thermal noise with another periodic or stochastic force [4]. In those cases all moments of the distribution of the noisy force do exist, and the existence of moments of the distribution of the particle's velocity is guaranteed. There are at least two reasons to consider the directed motion of a particle in a periodic ratchet potential under the influence of a heavy-tailed noise causing anomalously large particle displacements. One of them is pursuing the line of investigations of rectification of non-thermal noises and generalizing the corresponding considerations to the heavy-tailed cases which were found to be quite abundant under non-equilibrium conditions $[6,67,[8,9]$. Another one, connected with the former, is seeking for the way to characterize the ensuing directed motion in the case when the corresponding statistical moments are absent, so that neither the dispersion nor even the mean of the corresponding displacements exist. In consequence, in such cases the standard characteristics of motion like

\footnotetext{
*Electronic address: bartek@th.if.uj.edu.pl

${ }^{\dagger}$ Electronic address: gudowska@th.if.uj.edu.pl

${ }_{\ddagger}^{\ddagger}$ Electronic address: igor.sokolov@physik.hu-berlin.de
}

mean velocity or Peclet number become inapplicable.

The interplay of deterministic dynamics and perturbative Lévy-type noises have been addressed in literature in various scenarios including several noise-induced effects like resonant activation [10, 11], stochastic resonance [12], dynamical hysteresis [12, 13], studies of decay/relaxation properties of the probability densities [14, 15], escape from bounded intervals 16, 17], the classical barrier crossing problem 18, 19, 20] or examination of stationary states [15, 21, 22, 23]. However, very few examples 24 tackle the problem of Lévy noise driven dynamics of periodic systems.

In the present work we study the behavior of a particle in the periodic potential with a broken spatial symmetry subjected to the action of a symmetric Lévy stable noise. The $\alpha$-stable noise can be expected to occur in systems being out of thermal equilibrium, where detailed balance conditions is violated. In such realms one can anticipate noise induced current in a static potential with broken spatial symmetry. Consequently, what we address here as the Lévy ratchet resembles so called thermal ratchet [4] with time-dependent temperature variations. Heavy tails of distribution of additive stochastic increments lead however to considerable peculiarities of such motion, due to power-law character of probability densities of the particle's displacements, which lack the dispersion and may also lack the mean. Therefore, the examination of the current defined as the time derivative of the mean position may not be adequate for the Lévy ratchet, and one has to look for other quantities which characterize the motion caused by the interplay of the noise and a potential. Instead of examination of the current and of the effective diffusion coefficient we 
discuss several robust probabilistic characteristics of the particle's displacement based on its cumulative distribution: the behavior of median, which allows to introduce the group velocity, the growth of interquantile distances and the behavior of splitting probabilities (as defined in Sec. IIID).

As it will be discussed futher on, symmetric stable noise acting in a presence of a potential with broken spatial symmetry can induce directed motion of a test particle in an overdamped system. As long as the mean value of the perturbative noise exists and equals zero the displacement of the particle also possesses a mean. The overall motion can be then characterized by the temporal changes in the mean position, or, in the other words, by a mean velocity. In the case of an ensemble of particles mean velocity corresponds to the overall current, which is an adequate measure of the directionality of motion [4]. This situation is realized for $\alpha$-stable noises with $1<\alpha<2$.

If the noise, however, is distributed according to a Lévy-stable law with $0<\alpha<1$, neither the mean of the noise nor the mean of the overall displacement exist, so that one has to look for other quantities characterizing the directed motion. The discussion of these quantities, parallelly to investigation of the properties of the directed transport, is the main topic of the present article. Its structure is as follows. The next section (Section III) presents the model under consideration. The obtained numerical results are included in Section III which is devoted to various characteristics of the considered ratcheting device. The cumulative distribution of the overall displacement probability is examined in Section IIA. whereas its main features like median allowing for a definition of a group velocity of the probability packet are studied in Section IIIB We discuss also interquantile distance (Section IIIC), as well as splitting probability (Section IIID) that defines the fraction of current going to the left versus right direction when leaving an interval of finite length. Finally, (Sec. IIIE) we discuss properties of the first escape time distribution from a box of given width. The paper is closed with summary and concluding remarks (Section IV]).

\section{MODEL}

In what follows we consider the system described by the overdamped Langevin equation

$$
\frac{d x}{d t}=-V^{\prime}(x)+\zeta(t)
$$

where $\zeta(t)$ stands for a Lévy stable noise, i.e. the noise for which increments are distributed according to a stable probability density. Finally, $V(x)$ is an external static ratchet potential, see Fig. 1, with broken spatial symmetry

$$
V(x)=\frac{1}{2 \pi}\left[\sin \frac{2 \pi x}{L}+\frac{1}{4} \sin \frac{4 \pi x}{L}\right]
$$

Initially, at $t=0$, a test particle is located at $x=0$.

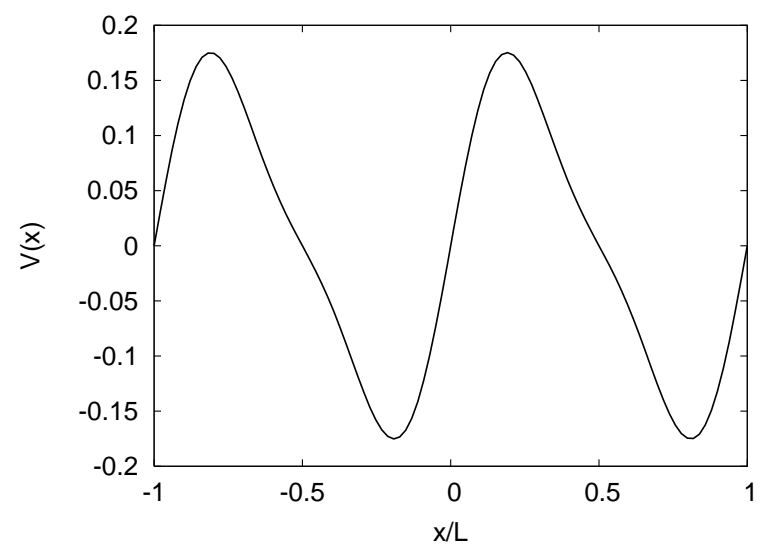

FIG. 1: The potential, $V(x)$, with broken spatial symmetry given by Eq. (2) used for examination of the studied Lévy ratchet.

In the most general case Lévy distributions correspond to a 4-parameters family of the probability density functions [25, 26, 27]. Here we restrict studies to symmetric stable noises, $L_{\alpha}(\zeta ; \sigma, \mu)$, which are characterized by the Fourier-transform $\phi(k)=\int_{-\infty}^{\infty} e^{i k \zeta} L_{\alpha}(\zeta ; \sigma, \mu) d \zeta$ of the probability density given by [25, 26, 27]

$$
\phi(k)=\exp \left[i k \mu-\sigma^{\alpha}|k|^{\alpha}\right] .
$$

The parameter $\alpha$ (where $\alpha \in(0,2])$ is the stability index of the distribution describing (for $\alpha<2$ ) its asymptotic "fat" tail characteristics yielding $L_{\alpha}(\zeta ; \sigma, \mu) \sim|\zeta|^{-(1+\alpha)}$ for large $\zeta$. The parameter $\mu$ denotes the location parameter representing position of the modal value. The Gaussian distribution, see Eq. (3), corresponds to a special case of a Lévy law for $\alpha=2$, with $\mu$ interpreted now as a mean and $\sigma$ as the dispersion of the distribution. Additionally, we assume $\mu=0$ and $\sigma=\{0.25,0.5,1\}$, i.e. we consider symmetric, strictly stable noises only. It is worthy mentioning that nonzero asymmetry parameter, which is not considered here, introduces a preferred direction of noise pulses and consequently may lead to a preferred direction of motion [23, 24].

\section{RESULTS}

For symmetric Lévy noises, Eq. (11) is equivalent to the following fractional differential Fokker-Planck equation [28, 29, 30]

$$
\frac{\partial P(x, t)}{\partial t}=\frac{\partial}{\partial x} V^{\prime}(x) P(x, t)+\sigma^{\alpha} \frac{\partial^{\alpha} P(x, t)}{\partial|x|^{\alpha}},
$$

where $\alpha$ and $\sigma$ characterize the noise. The fractional (Riesz-Weyl) derivative is interpreted in the sense of the Fourier transform [21, 22, 31] $\frac{\partial^{\alpha}}{\partial|x|^{\alpha}} f(x)=$ 
$-\int_{-\infty}^{\infty} \frac{d k}{2 \pi} e^{-i k x}|k|^{\alpha} \hat{f}(k)$. Nevertheless, due to possible instabilities [32] of numerical approximations to Eq. (44) 33] in the following studies we use approach based solely on the Langevin equation (1).

The results presented below were obtained by the numerical integration of Eq. (11) with Lévy stable noises using the methods discussed in Refs. [16, 20, 26, 27]. The numerical integration of Eq. (11) was performed with the time step of $\Delta t=10^{-3}$. The number of realizations varied from $N=10^{5}$ to $N=10^{6}$ leading in all cases to consistent results.

Initially, a test particle is located at $x=0$. In course of time the distribution of the particle's positions $x$ evolves due to the presence of the stochastic force and of the deterministic potential in Eq. (1). As we proceed to show, the ensuing coarse-grained probability distribution of $x$ is a symmetric distribution of the power-law type (Sec. IIIA). The overall motion of the probability density is characterized by changes in the location of its median (Sec. IIIB). The width of the probability density grows with time. The growing width of the distribution is characterized by growing interquantile distance (Sec. IIIC). Still another characteristic of the motion can be addressed by analyzing behavior of splitting probabilities: A particle initially located at $x=0$ can escape from an interval of given width $L$ centered at the initial position to the left or to the right. The statistics of the corresponding escape events (splitting probabilities, Sec. IIID) provides another measure of the directionality of motion. Finally, mean first escape time provides a characteristic of the time scales involved in such escapes (Sec. IIIE).

\section{A. Cumulative distribution}

We first discuss the cumulative distribution function (CDF) of the particle's displacement under the non-equilibrium additive noise. The $\mathrm{CDF}$ is given by $F(x, t)=\int_{-\infty}^{x} P\left(x^{\prime}, t\right) d x^{\prime}$ with $P(x, t)$ being the corresponding probability density function (PDF), which can be interpreted in terms of particle concentration. Simulations show, that in course of time the corresponding PDF attains a symmetric form, see Fig. 2. The density $P(x, t)$ is then found to move in one direction and to broaden in time. These effects can be seen by inspection of the overall position of the particles characterized by the median of the distribution $q_{0.5}$ (cf. Fig. 5) and its width defined by the interquantile distance, e.g. $q_{0.8}(t)-q_{0.2}(t)$ (cf. Fig. 7). The quantiles $q_{m}(0<m<1)$ are defined according to the relation $F\left(q_{m}, t\right)=m$.

For symmetric probability densities, i.e. densities such that $P\left(q_{0.5}-x, t\right)=P\left(x+q_{0.5}, t\right)$ the cumulative density $F(x, t)$ fulfills

$$
F(x, t)=1-F\left(2 q_{0.5}-x, t\right) .
$$

For the Lévy ratchet system, the constructed CDFs prove that PDF recorded at given time are symmetric with re- spect to median $\left(q_{0.5}\right)$ if the space scales much larger than the period of the potential are considered, see Fig. 1 and 2
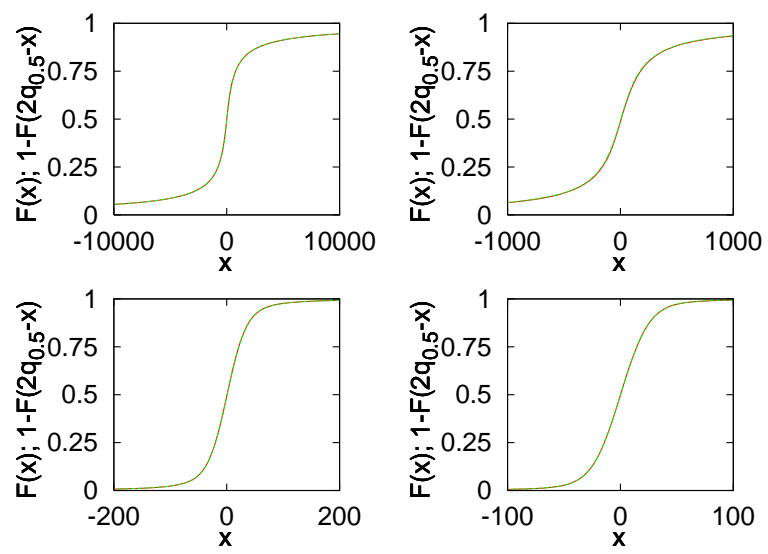

FIG. 2: (Color online) Cumulative distributions $F(x, t=100)$ and $1-F\left(2 q_{0.5}-x, t=100\right)$ for $\alpha=0.7$ (top left panel), $\alpha=0.9$ (top right panel), $\alpha=1.5$ (bottom left panel), $\alpha=1.7$ (bottom right panel) with $\sigma=1$. The perfect agreement between both curves indicates the symmetric shape of corresponding PDFs with respect to medians. The same agreement is observed for smaller values of the noise intensity $\sigma$. Note the large difference in scales for different values of $\alpha$.

The distributions of the particles' displacements in Fig. 2 exhibit power-law tails. To detect them it is enough to consider the asymptotic behavior of the 'survival probability' $G(x, t)=1-F(x, t)$ for large $x$, see Fig. 3 clearly displaying this power-law behavior. The simplest hypothesis here would be that the corresponding exponent is the same as the one of the noise; if $\zeta(t)$ has a stable density characterized by exponent $\alpha$, i.e. $P(\zeta, t) \propto \zeta^{-(1+\alpha)}$ for large $\zeta$, the 'survival probability' should behave like $G(x, t) \propto x^{\gamma}$ with $\gamma=\alpha$. This kind of behavior is indeed observed for the values of stability index $\alpha$ smaller or around one, see Fig. 4 . For larger $\alpha, G(x, t)$ still shows a power-law asymptotic, see Fig. 3 , however, the numerically obtained values of exponents $\gamma$ differ from $\alpha$, see Fig. 4. This difference is pertinent to a very far tail of the distribution, since the behavior of the interquantile distances, say $q_{0.2}(t)-q_{0.8}(t)$, unveils the behavior compatible with the $\gamma=\alpha$ assumption for all $\alpha$, see Sec. IIIC.

Inspection of 'survival probability' $G(x, t)$, see Fig. 3, suggests that for small value of $\alpha$ the particle practically does not feel the potential. Therefore, the particle behaves like a free particle and the exponent characterizing 'survival probability' is the same as the one characterizing statistical properties of the noise. This observation is in accordance with predictions of the continuous time random walks theory in situations when the mean waiting time for a next jump is finite [34, 35]. For increasing values of $\alpha$, the motion gets to be more sensitive to the structure of the potential. Consequently, the form of 
$G(x, t)$ in the intermediate range of $x$ diverges from the pure Lévy distribution. The difference between $\gamma$ and $\alpha$ is probably explained by extremely slow convergence of the tail of the corresponding distribution to its asymptotic form.

In order to fit the tail asymptotics properly, it is necessary to have large statistics corresponding to extreme events which should cover at least a couple of orders of magnitude. Such a coverage is rather easily reached for values of $\alpha \leqslant 1.1$. However, for large values of the stability index $\alpha(\alpha>1.1)$, the statistics of 'survival probability' $G(x, t)$ for very large values of $x$ is quite poor: the exploration of large $x$ dependence requires simulation times which are beyond our possibilities. The values of $\gamma$ obtained in simulations did not indicate changes for chosen simulation times, however the fitted slope was found to be sensitive to the choice of the threshold from which a power-law is fitted.

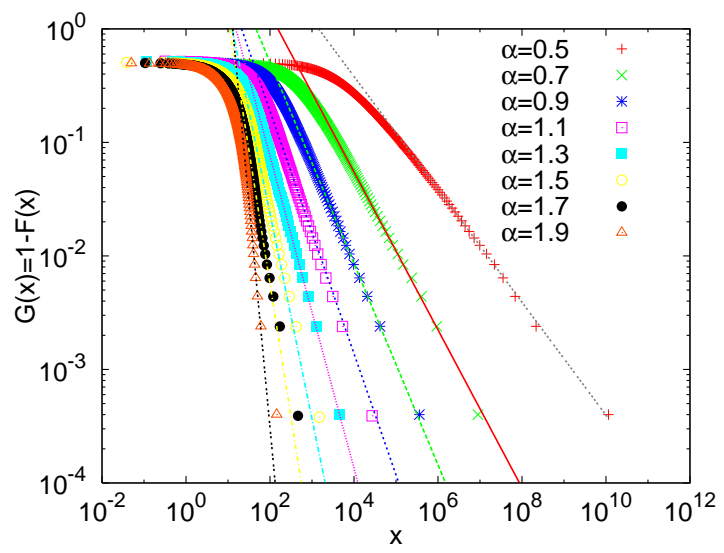

FIG. 3: (Color online) 'Survival probability' $G(x, t=100)=$ $1-F(x, t=100)$ with $\sigma=1$. For large $x$, numerical simulations demonstrate power-law character of 'survival probabilities' $G(x, t=100)$. Nevertheless, the value of the exponent characterizing large $x$ behavior is different from $\alpha$ when underlying noise is characterized by $\alpha \geqslant 1.3$, see Fig. 4

\section{B. Median and group velocity}

The position of the median describes the overall motion of the probability density to find a particle in the vicinity of $x$. In our case, the corresponding PDF coarsegrained over the period of the potential, is a symmetric, monomodal function, so that the temporal change of the median (which coincides with the maximum of the PDF) gives us the possibility to define the group velocity of the particles' packet as $v=d q_{0.5} / d t$. Our numerical simulations show that the position of median $q_{0.5}(t)$ changes linearly with time

$$
q_{0.5}(t)=v \cdot t+b,
$$

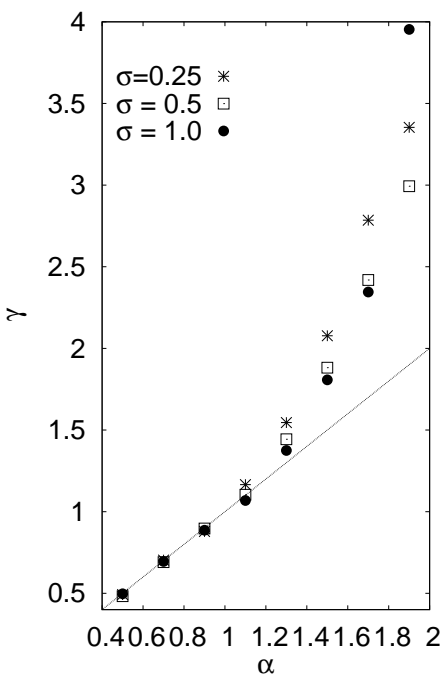

FIG. 4: Value of the exponent $\gamma$ in 'survival probability' describing the asymptotic behavior of its tails $G(x, t) \propto x^{\gamma}$, see Fig. 3 For $\alpha \geqslant 1.3$, the exponent characterizing tails' asymptotic differs from the index $\alpha$ of the underlying noise because the motion of the test particle starts to be more sensitive to the structure of the potential. Relative errors of the fit are smaller than $1 \%$ of value of exponents. Consequently, error bars are smaller than symbol size.

which corresponds to the constant group velocity. Note that this group velocity can be defined even in the case when the mean velocity or current does not exist due to large fluctuations, and defines the behavior of the typical displacement of particles with time. Fig. 5 presents location of the median as a function of time for different values of stability exponents $\alpha$. The 'periodic-like' modulation of the median position is caused by the periodic shape of the potential, see ordinate of Fig. 1. For the constant amplitude of the noise the group velocity decreases with increase of the exponent $\alpha$ and disappears in the Gaussian case $\alpha=2$, see Fig. 6, in which case the noise satisfies the detailed balance condition.

The 'survival probability' shows that asymptotic behavior of the probability density of a particle position is not sensitive to the detailed structure of the potential. The presence of potential is visible for larger values of $\alpha$ when the exponent characterizing the tails of distributions starts to differ from $\alpha$, see Figs. 3 and 4, Different situation takes place for median which reflects the structure of the potential. It is manifested in the periodic modulation of median position visible in Fig. 5 This periodic structure has the same period like the potential. This suggests that tails of distributions can freely overpass the potential maxima, while the motion of median is strongly affected by presence of maxima of the potential. 


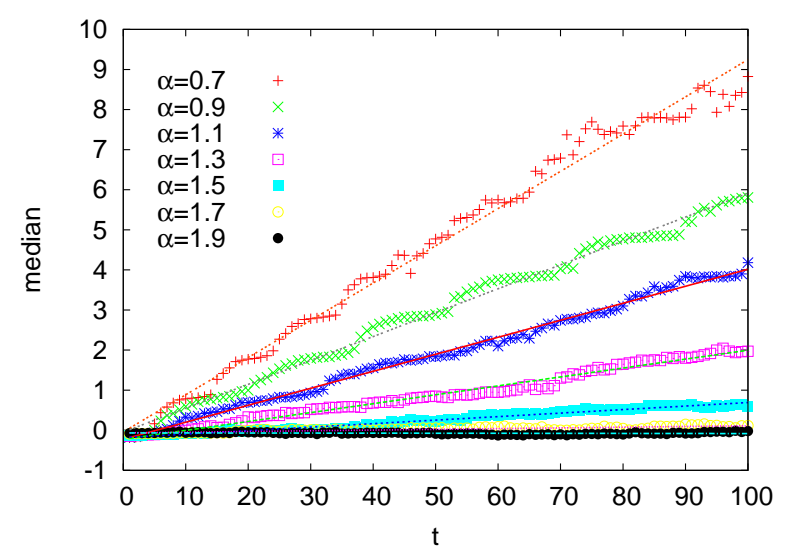

FIG. 5: (Color online) Time dependence of the location of median, $q_{0.5}(t)$ which describes the overall motion of the ensemble of particles injected into the Lévy ratchet at $t=0$ $(\sigma=1)$. The linear growth of median with time allows to define the group velocity of the packet of particles.

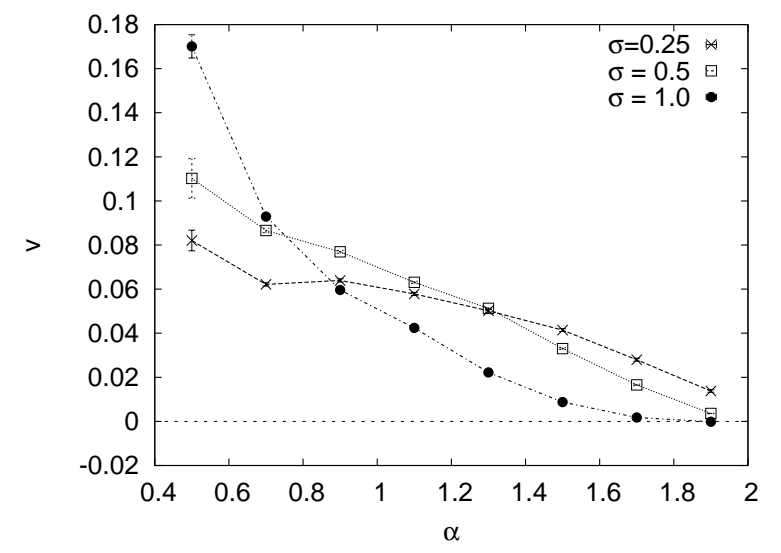

FIG. 6: Value of the group velocity (coefficient $v$ in Eq. (6) characterizing slope of curves in Fig. 5) as a function of the stability index $\alpha$. The lines are drawn to guide the eye only. The fastest overall motion of the probability density is observed for small values of stability index $\alpha$ for which large noise increments are more probable. The value of $v$ vanishes towards $\alpha=2$ corresponding to the equilibrium Gaussian noise for which persistent current disappears. Error bars represent errors of the fit. Error bars, except for those for $\alpha=0.5$, are smaller than symbol size.

\section{Interquantile distance}

Interquantile distance is a robust measure characterizing the distribution's width even in cases when moments of the distribution do not exist. For the studied Lévy ratchet this interquantile distance scales like $t^{p}$ independently of the value of the noise intensity $\sigma$, i.e.

$$
q_{m}(t)-q_{1-m}(t) \propto t^{p}
$$

where $p=1 / \alpha$. Fig. 7 presents time dependence of interquantile distance $q_{0.2}(t)-q_{0.8}(t)$ along with fitted curves. Fig. 8 presents values of fitted exponents, together with the predicted $1 / \alpha$-behavior. The interquantile distance $q_{m}(t)-q_{1-m}(t)$ scales in the same manner also for other values of $m(0<m<1)$. Note that the periodic modulation which is typical for evolution of median, see Fig. [5 can be not visible for interquantile distance. Essentially, this modulation does not appear in the behavior of whatever quantiles $q_{m}(t)$ with $m \neq 0.5$ and sufficiently large noise intensity $\sigma$. The decrease of the noise intensity $\sigma$, introduces periodic modulation also to interquantile distance which can be the most easily observed for large values of the stability index $\alpha$ (figure not shown).

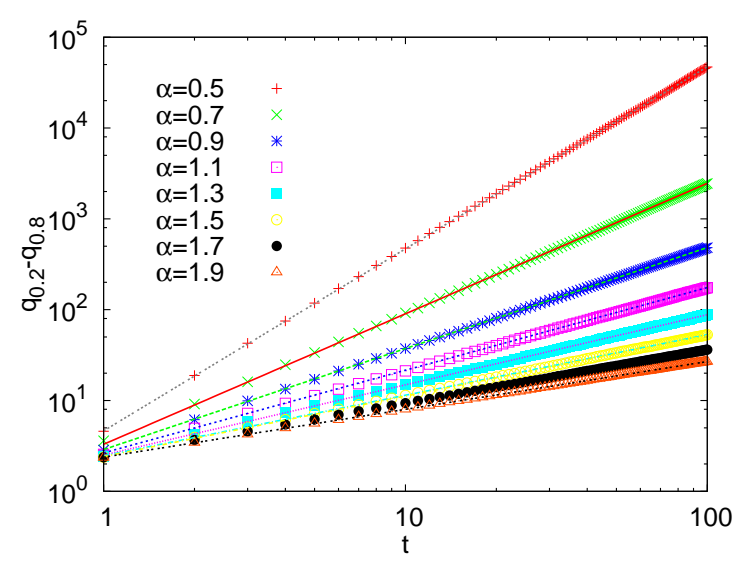

FIG. 7: (Color online) Time dependence of the interquantile distance $q_{0.2}(t)-q_{0.8}(t)$ with $\sigma=1$ together with fitted theoretical curves. The decrease of scale parameter $\sigma$ makes the particle more sensitive to the shape of the potential and consequently introduces periodic-like modulation of interquantile distances (results not shown).

\section{Splitting probability}

Considering splitting probabilities gives us another characteristics of the directionality of motion. Splitting probability is a measure of the preferred direction to which the first escape from the system takes place. In this case we consider an interval of length $L$ centered around the point where particles are introduced, and consider the probability for the particle to escape the interval through its left (right) boundary. Fig. 9 presents probability $\pi_{L}$ of the first escape to left as a function of the box halfwidth. The presented splitting probability indicates that the preferred direction of the first escape from the system is to the right, since for longer intervals $\pi_{L}<1 / 2$. Furthermore, decrease of the scale parameter $\sigma$ makes this tendency stronger.

Fig. 9 shows a clear difference between the cases $1<$ $\alpha<2$ and $\alpha<1$. In the first case for the intervals 


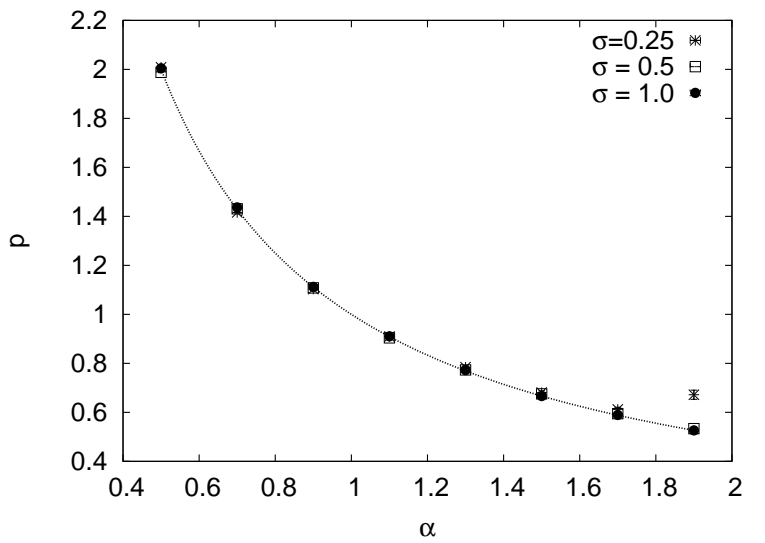

FIG. 8: Value of the exponent $p$ in Eq. (7) as a function of the stability index $\alpha$. Line presents theoretical dependence of the exponent characterizing growth of the interquantile distance, i.e. $1 / \alpha$. Error bars are smaller than symbol size.
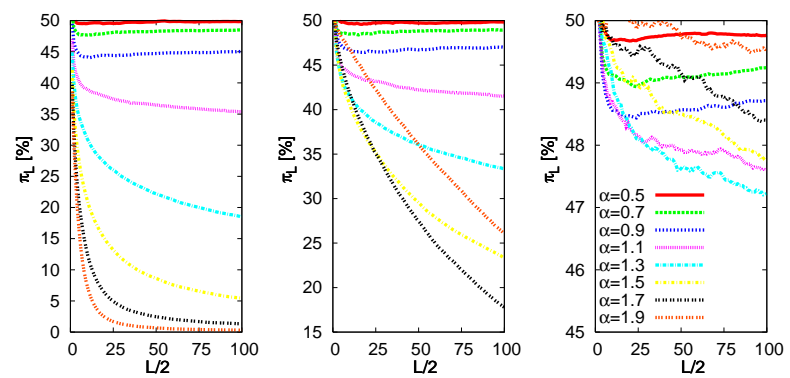

FIG. 9: (Color online) Splitting probability, $\pi_{L}$, i.e. probability of the first escape to the left as a function of the box half-width. Initially particle is located in the middle of the box of half-width $L / 2$, see Eq. (2) and Fig. 11 Various panels correspond to different values of the scale parameter $\sigma$ : $\sigma=0.25$ (left panel), $\sigma=0.5$ (middle panel) and $\sigma=1$ (right panel).

("boxes") much larger than the period of the potential the probability $\pi_{L}$ decays monotonically with the box size, which means than more and more particles leave the box through its right boundary. In the second case, as exemplified by $\alpha=0.9$ the behavior of $\pi_{L}$ is nonmomotonical: it decays with $L$ up to $L / 2 \approx 20$ and then starts to grow again.

This striking difference between the results for $\alpha>1$ and ones for $\alpha<1$ gets clear when returning to the behavior of the interquantile distances. In the first case the shift of the distribution's median (coinciding with its maximum), going linearly in time, is asymptotically faster than the growth of the distribution's width, which allows us to discuss the position of the typical particle as a mean position and to introduce the mean current. In course of the time (necessary to leave the larger box) the typical displacement wins over the spread, and most of the particles come out of the box through its one (right) boundary.

In the case $\alpha<1$ the width of the distribution grows faster than its maximum moves. Due to the wellprescribed initial position, the contribution of the overall motion is still perceptible at shorter times and thus for smaller boxes, and the difference between the probabilities to leave the box through its left and right boundary grows. At longer times the broadening of the distribution prevails, typical displacement gets hardly relevant on the background of fluctuations, and the difference in splitting probabilities decays. However, the distribution still has a pronounced maximum, and its motion defines the group velocity.

\section{E. Exit Time}

The spiting probability is a measure of directionality of the first escape from a box of a given width. The quantity which is very closely related to the spiting probability is exit time, i.e. the time which is needed to escape from the same box. Here we study escape time, $\tau$, which is the first escape time from the box through one of its edges, i.e. we do not distinguish between borders of the box.

The same information which is included in the escape time distribution is included in the survival probability. Survival probability, $S(t)=1-F(t)$, is the probability of finding a particle in the box of given width at time $t$, i.e. it is the ratio of particles which at time $t$ are still in the box. The survival probability $S(t)$ is exponential function of time [16, 20, 36]. In the Fig. [10, survival probabilities for $L=10$ are presented with $\sigma=1$. Results for other values of the box width $L$ and scale parameter $\sigma$ also show exponential behavior.

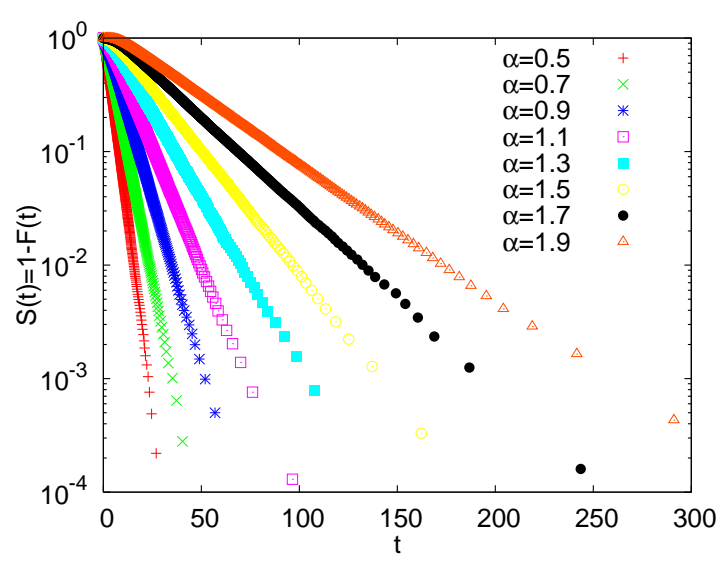

FIG. 10: (Color online) Survival probability, $S(t)=1-F(t)$, i.e. the probability of finding a particle in the box of the width $L=10$ for $\sigma=1$. Survival probabilities are of the exponential type regardless of the value of $L$ and $\sigma$.

From first escape time distribution it is possible to calculate the mean exit time. For a free particle starting its 


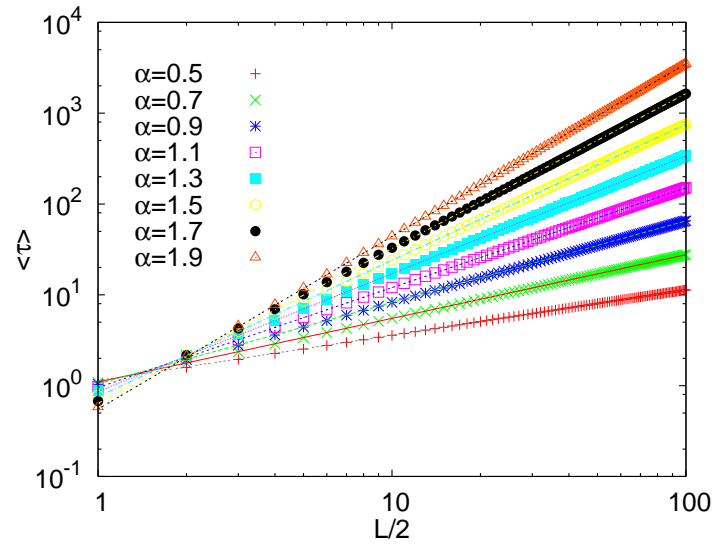

FIG. 11: (Color online) Mean exit time, $\langle\tau\rangle$, i.e. the average of the first escape time from the box as a function of the box half-width for $\sigma=1$. Initially a particle is located in the middle of the box of the half-width $L / 2$, see Eq. (2) and Fig. 1

motion in the middle of the interval of width $L$ the mean exit time is [17]

$$
\langle\tau(x(0)=0)\rangle=\frac{(L / 2)^{\alpha}}{\sigma^{\alpha} \Gamma(\alpha+1)}=a \cdot\left(\frac{L}{2}\right)^{b} .
$$

From Eq. (8) it implies that $\langle\tau\rangle \propto L^{\alpha}$. The same kind of behavior can be observed for the Lévy ratchet. For the large value of the scale parameter $\sigma$, the presence of the potential does not affect the dynamics of the particle. Consequently, both the prefactor and exponent are like in the free case, see Eq. (8). The decrease of the noise intensity affects $L^{\alpha}$ scaling and the prefactor in Eq. (8), especially in situations when the Lévy noise is close to the Gaussian one and small values of the scale parameter $\sigma$ are considered. Nevertheless, for small to moderate values of the stability index $\alpha$, with all considered values of noise intensity $\sigma$, the first escape time is like in the free case. Mean exit time as a function of the box halfwidth with $\sigma=1$ is depicted in Fig. 11. Fig. 12 tests applicability of Eq. (8).

\section{SUMMARY AND CONCLUSIONS}

Alltogether, we have numerically documented, that the motion of the overdamped particle subjected to a symmetric Lévy noise in a potential with a broken spatial symmetry leads to occurrence of directionality of motion. The preferred direction of motion results as a consequence of an interplay between non equilibrium character of the underlying stable noise and the broken spatial symmetry of the static potential. However, the characteristics of the particle's displacement, like mean velocity and the diffusion coefficient, describing the particle's dispersion, get inappropriate when white Lévy noises are
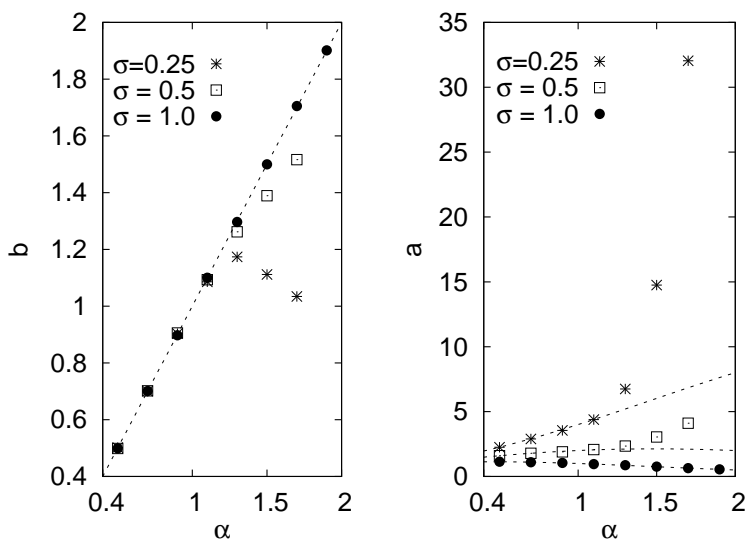

FIG. 12: Value of the exponent $b$ and the prefactor $a$ in Eq. (8) as a function of the stability index $\alpha$. Lines present theoretical values of the exponent and the prefactor characterizing mean escape time for a free particle, i.e. $\alpha$ and $1 /\left[\Gamma(\alpha+1) \sigma^{\alpha}\right]$ respectively.

considered. Diverging variance and possibly non-existing mean make it necessary to introduce other characteristics of the directed motion. Consequently, the conducted research is based on robust measures characterizing the collective motion of Lévy-Brownian particles, like group velocity, interquantile width of the displacements' distribution and splitting probabilities.

In the system under the study, the initially sharp distribution of particles' positions broadens in course of time leading to symmetric probability density of the powerlaw type. The median of the distribution, which characterizes the group velocity of the particle packet, moves linearly with time. The fastest motion of median is observed for small values of the stability exponent $\alpha$. The coherence of the motion can be characterized by the interquantile width of the distribution of the particles' position, whose growth with time was found to follows the $q_{m}(t)-q_{1-m}(t) \propto t^{1 / \alpha}$ pattern. For $\alpha>1$ the width of the distribution grows therefore slower than its maximum moves, contrary to the situation when $0<\alpha<1$ and the opposite is the case. This fact is mirrored in the behavior of splitting probabilities for leaving an interval of a given length.

Eq. (11) describes the dynamics of the Lévy ratchet for any value of the noise parameters. Here, we restricted our studies to symmetric noises only. In such a case the only relevant noise parameter is the stability index $\alpha$ which characterizes the asymptotic power law behavior of additive noise pulses. The stability index controls the level of non-equilibrity of the noise. On the one hand, for values of $\alpha$ smaller than 2, the Lévy noise serves as a prototypical perturbation source that drives the system out of equilibrium. On the other hand, for $\alpha=2$, the Lévy noise becomes Gaussian noise and models thermal fluctuations in the vicinity of the equilibrium state. The rectification, ratcheting effect is visible only for values of 
$\alpha<2$. The increase of the stability index $\alpha$ decreases the ratcheting effect. Finally for $\alpha=2$, similarly to the original ratchet and pawl device [4], the absence of the average particles' current is a simple consequence of the second law of thermodynamics: despite the broken spatial symmetry assured by the form of the potential, no systematic preferential motion of the random dynamics can be detected and the ratcheting effect disappears.

The emergence of the rectification in the "minimal Lévy ratchet" (Eq. (1)) follows as a result of the nonequilibrium type of the stochastic driving and broken spatial symmetry of the potential. On the one hand, the ratcheting effect is observable for symmetric noises, therefore it is induced by the potential. On the other hand, in the minimal Lévy ratchet scenario the presence of the potential is not always explicit: For a free Lévy particle the probability density of particle positions is given by a Lévy distribution with growing width and the same tails characteristics as the underlying noise. The same effect is observed here for the Lévy ratchet with $\alpha<1.3$ when the tail asymptotic of the PDF for a particle position becomes characterized by the same exponent like the underlying noise. For $\alpha \geqslant 1.3$ these exponents start to differ suggesting that only for $\alpha$ s excessing a certain threshold value the particle starts to feel the potential. On the contrary, closer examination of the median reveals periodic structure of the median position with the same space period as the one characterizing the potential. Finally, for all values of the stability index $\alpha$ with sufficiently large noise intensity $\sigma$, values of the mean exit time scale as in the free particle case. The decrease of the scale parameter $\sigma$ makes the system more sensitive to the presence of a potential.

For a Lévy ratchet, symmetric stable noise together with periodic potential with broken spatial symmetry provide a minimal setup for the occurrence of directed current. Contrary to Gaussian noises, stable Lévy ones can be intrinsically asymmetric; the asymmetry of the noise introduce preferred direction of the noise pulses might affect the current. On the other hand, the asymmetry of noise breaks symmetry of the system and introduces a component which is of 'non-zero average type.' The asymmetric Lévy noise can be still incorporated to the Lévy ratchet in the 'zero-average manner.' This can be reached by making asymmetry parameter a periodic function of time [13]. The time dependence of asymmetry parameter will slightly modify the type of the Lévy ratchet making it closer to the ones of either 'thermal' or 'tilting' type. Considering such situations might be an interesting further step pursuing our line of investigations.

\section{Acknowledgments}

Authors acknowledge stimulating and inspiring discussions with E. Barkai and I. Pavlyukevich. The research has been supported by the Marie Curie TOK COCOS grant (6th EU Framework Program under Contract No. MTKD-CT-2004-517186) and European Science Foundation (ESF) via 'Stochastic Dynamics: fundamentals and applications' (STOCHDYN) program. Additionally, BD acknowledges the support from the Foundation for Polish Science and the hospitality of the Humboldt University of Berlin and the Niels Bohr Institute (Copenhagen). The support by DFG within SFB555 is also acknowledged.
[1] R. D. Astumian and M. Bier, Phys. Rev. Lett. 72, 1766 (1994).

[2] M. O. Magnasco, Phys. Rev. Lett. 71, 1477 (1994).

[3] J. Kula, T. Czernik, and J. Łuczka, Phys. Rev. Lett. 80, 1377 (1998).

[4] P. Reimann, Phys. Rep. 361, 57 (2002).

[5] P. Reimann and P. Hänggi, Appl. Phys. A 75, 169 (2002).

[6] Lévy Flights and Related Topics in Physics, edited by M. F. Shlesinger, G. M. Zaslavsky, and J. Frisch (Springer Verlag, Berlin, 1995).

[7] Lévy Processes: Theory and Applications, edited by O. E. Barndorff-Nielsen, T. Mikosch, and S. I. Resnick (Birkhäuser, Boston, 2001).

[8] A. A. Dubkov and B. Spagnolo, Fluct. and Noise Lett. 5, L267 (2005).

[9] A. A. Dubkov and B. Spagnolo, Acta Physica Polonica B 38, 1745 (2007).

[10] B. Dybiec and E. Gudowska-Nowak, Phys. Rev. E 69, 016105 (2004).

[11] B. Dybiec and E. Gudowska-Nowak, Fluct. and Noise Lett. 4, L273 (2004).

[12] B. Dybiec and E. Gudowska-Nowak, Acta Physica
Polonica B 37, 1479 (2006).

[13] B. Dybiec and E. Gudowska-Nowak, New J. Phys. 9, 452 (2007).

[14] B. Dybiec, Stochastic Resonant Effects in Systems Driven by Stable Noises, PhD Thesis, Faculty of Physics, Astronomy and Applied Computer Sciences, Marian Smoluchowski Institute of Physics, Jagellonian University, Kraków, Poland, 2005.

[15] A. V. Chechkin et al., J. Stat. Phys. 115, 1505 (2004).

[16] B. Dybiec, E. Gudowska-Nowak, and P. Hänggi, Phys. Rev. E 73, 046104 (2006).

[17] A. Zoia, A. Rosso, and M. Kardar, Phys. Rev. E 76, 21116 (2007).

[18] P. D. Ditlevsen, Phys. Rev. E 60, 172 (1999).

[19] A. Chechkin, V. Gonchar, J. Klafter, and R. Metzler, Europhys. Lett. 72, 348 (2005).

[20] B. Dybiec, E. Gudowska-Nowak, and P. Hänggi, Phys. Rev. E 75, 021109 (2007).

[21] A. V. Chechkin et al., Chem. Phys. 284, 233 (2002).

[22] A. V. Chechkin et al., Phys. Rev. E 67, 010102(R) (2003).

[23] B. Dybiec, E. Gudowska-Nowak, and I. M. Sokolov, Phys. 
Rev E 76, 041122 (2007).

[24] D. del Castillo-Negrete, V. Gonchar, and A. Chechkin, eprint arXiv: 0710.0883 (2007).

[25] W. Feller, An introduction to Probability Theory and its Applications (John Wiley, New York, 1968).

[26] A. Janicki and A. Weron, Simulation and Chaotic Behavior of $\alpha$-Stable Stochastic Processes (Marcel Dekker, New York, 1994).

[27] A. Janicki, Numerical and Statistical Approximation of Stochastic Differential Equations with Non-Gaussian Measures (Hugo Steinhaus Centre for Stochastic Methods, Wrocław, 1996).

[28] R. Metzler, E. Barkai, and J. Klafter, Europhys. Lett. 46, 431 (1999).
[29] V. V. Yanovsky, A. V. Chechkin, D. Schertzer, and A. V. Tur, Physica A 282, 13 (2000).

[30] D. Schertzer et al., J. Math. Phys. 42, 200 (2001).

[31] S. Jespersen, R. Metzler, and H. C. Fogedby, Phys. Rev. E 59, 2736 (1999).

[32] M. M. Meerschaert and C. Tadjeran, J. Comp. App. Math. 172, 65 (2004).

[33] R. Gorenflo et al., Chem. Phys. 284, 521 (2002).

[34] R. Metzler and J. Klafter, Phys. Rep. 339, 1 (2000).

[35] R. Metzler and J. Klafter, J. Phys. A: Math. Gen. 37, R161 (2004).

[36] P. Imkeller and I. Pavlyukevich, Stoch. Proc. and their Appl. 116, 611 (2006). 